\title{
Anatomical variations of Peroneus tertius and Extensor digitorum longus muscles: Cadaveric Study
}

\author{
Kosgallana $\mathrm{EW}^{1} *$, Eshwara $\mathrm{JM}^{1}$, Dissanayake $\mathrm{JK}^{1}$ \\ ${ }^{\prime}$ Department of Anatomy, Faculty of Medicine, University of Peradeniya. \\ *warukos@gmail.com
}

Peroneus tertius (PT) and extensor

digitorum longus (EDL), are muscles of the anterior compartment of the leg, important for the bipedal gait in humans. They are used as anatomical boundaries during arthroscopy of the ankle joint and widely used for soft tissue replacement surgeries. PT is implicated in the pathophysiology of Jones fracture of the fifth metatarsal bone. It was the objective of this project to study the characteristics of PT and EDL in cadaveric specimens. The PT and EDL muscles of the leg in 25 lower limb specimens were examined for features with regard to muscle length, tendon length, mid circumference, origin and insertion. A single muscle belly replacing PT and EDL was observed in 7 specimens giving rise to 5 tendons which had distal attachments to the lateral 4 digits of the foot and the base of $5^{\text {th }}$ metatarsal. The average circumference of these single muscles was $4.18 \mathrm{~cm}$. In the specimens where PT and EDL were separate, their average circumferences were $3.46 \mathrm{~cm}$ and $4.5 \mathrm{~cm}$ respectively. Distal insertion of the PT had variable attachments to the base and shaft of the fifth metatarsal bone and the fourth interosseous space. Further, in one cadaver, PT and EHL appeared to be attached closely together by a common fascial layer in between them, which extended to enclose the lower most fibers of the PT. Correct identification of individual anatomy of these muscles has important surgical implications. Susceptibility to Jones fracture may vary with the variability of PT especially with regard to its insertion. 